PARSONS, P. A. 1966. The genotypic control of longevity in Drosophila melanogaster under two environmental regimes. Aust. 7. Biol. Sci., 19, 587-591.

PARsons, P. A. 1970. Genetic heterogeneity in natural populations of Drosophila melanogaster for ability to withstand desiccation. Theoret. and Appl. Genetics, 40, 261-266.

PARsons, P. A., AND KAUl, D. 1966. Mating speed and duration of copulation in Drosophila pseudoobscura. Heredity, 21, 219-225.

PARSONS, P. A., AND maKenZIE, J. A. 1971. The ecological genetics of Drosophila. Evolutionary Biology (in press).

POWELL, J. R., AND RICHMOND, R. C. 1971. Evidence of heterosis associated with an enzyme locus in a natural population of Drosophila. Genetics, (in press).

PRAKASH, s., AND LEWONTIN, R. G. 1968. A molecular approach to the study of genic heterozygosity in natural populations. III. Direct evidence of coadaptation in gene arrangements of Drosophila. Proc. Natl. Acad. Sci. U.S., 59, 398-405.

STONE, w. s., KOJIMA, K-I., AND JOHnson, F. M. 1969. Enzyme polymorphisms in animal populations. Japan 7 . Genetics, 44, Suppl. 1, 166-171.

тномson, J. A. 1964. An experimental study of the influence of environmental factors on population structure in Drosophila pseudoobscura. Forlowa. Ph.D. Thesis, University of Melbourne.

TOBARI, I. 1966. Effects of temperature on the viabilities of homozygotes and heterozygotes for second chromosomes of Drosophila melanogaster. Genetics, 54, 783-791.

VAN VALEN, L., LEVINE, L., AND BEARDMORE, J. A. 1962. Temperature sensitivity of chromosomal polymorphism in Drosophila pseudoobscura. Genetica, 33, 113-127.

WRIGHT, s., AND DOBZHANSKy, TH. 1946. Genetics of natural populations. XII. Experimental reproduction of some of the changes caused by natural selection in certain populations of Drosophila pseudoobscura. Genetics, 31, 125-156.

\title{
THE EFFECT OF UNEQUAL MATING FREQUENCIES ON THE VARIANCE EFFECTIVE NUMBER IN RANDOM MATING POPULATIONS
}

\author{
P. STAM \\ Department of Genetics of the Agricultural University, Wageningen, The Netherlands
}

Received 21.xii.70

THE effective number in a random mating population can be described as " inbreeding effective number" or, alternatively, as "variance effective number" (Crow, 1954; Grow and Kimura, 1970). The latter, $\mathcal{N} e(v)$, is defined by the variance of gene frequency change $(\Delta q)$ per generation:

where $p=1-q$.

$$
\operatorname{var}(\Delta q)=\frac{p q}{2 \mathcal{N} e(v)}
$$

Following Grow (1954), we have

$$
\operatorname{var}(\Delta q)=\frac{p q}{4 \mathcal{N}}\left(1+\frac{\operatorname{var}(k)}{\varepsilon(k)}\right)
$$

in which $\mathcal{N}$ is the actual population number and $k$ is the number of effective gametes contributed by a single individual. When $k$ is Poisson-distributed, $\operatorname{var}(k) / \varepsilon(k)$ equals unity and (2) reduces to the expression in the binomial sampling model:

$$
\operatorname{var}(\Delta q)=\frac{p q}{2 \mathcal{N}}
$$

so that $\mathcal{N} e(v)=\mathcal{N}$.

Now, individual variation in number of matings, whether or not in- 
fluenced by competition, leads via $\operatorname{var}(k) / \varepsilon(k)>1$ to $\mathcal{N} e(v)<\mathcal{N}$. Initially it was thought that this discrepancy, arising from multiple matings, could be rather large. However, Crow (1954) gives some empirical data for Drosophila males and females to show (see also Crow and Kimura, 1970) that the effective number and the actual number are at least not greatly different (table 1), the ratio of these two being approximately 0.75 for females and 0.50 for males.

TABLE 1

Relation of effective and actual population numbers in experimental data on Drosophila females and males. The adjusted values have been obtained by correcting to constant population size, under random survival of zygotes. Note that the adjusted ratio is larger for males than for females, which reflects a larger var $(\mathbf{k})$ for males. (From Crow, 1954)

\begin{tabular}{|c|c|c|c|c|}
\hline & $\varepsilon(k)$ & $\frac{\operatorname{var}(k)}{\varepsilon(k)}$ & $\begin{array}{c}\operatorname{var}(k) / \varepsilon(k) \\
\text { adjusted to } \\
\varepsilon(k)=2\end{array}$ & $\frac{\mathcal{N}_{e}(v)}{\mathcal{N}}$ \\
\hline \multicolumn{5}{|l|}{ Drosophila females } \\
\hline Adult progeny & $13 \cdot 9$ & $4 \cdot 73$ & 1.82 & 0.71 \\
\hline Egg laying ( 5 day) & $37 \cdot 2$ & $11 \cdot 45$ & 1.77 & 0.72 \\
\hline \multicolumn{5}{|l|}{ Eggs, lifetime } \\
\hline wild type, $25^{\circ}$ & 714 & 130 & $1 \cdot 36$ & $0 \cdot 85$ \\
\hline wild type, $30^{\circ}$ & 430 & 154 & 1.71 & 0.74 \\
\hline wild type, $19^{\circ}$ & 914 & 111 & 1.23 & 0.90 \\
\hline vestigial & 432 & 102 & 1.46 & 0.81 \\
\hline Drosophila males & 17.9 & $11 \cdot 35$ & $3 \cdot 18$ & $0 \cdot 48$ \\
\hline
\end{tabular}

In the following a realistic model will be proposed and an exact formula presented which accommodates $\mathcal{N} e(v)<\mathcal{N}$, resulting from individual variation in mating frequency.

Let, in a virginal population with equal number of both sexes, the variables $M_{f}$ and $M_{m}$ be the mating frequencies, in a given time interval of individual females and males respectively, and let $x_{f}$ and $x_{m}$ be the number of effective gametes contributed by these individual females and males per mating, that is their number of gametes.generating surviving offspring. Then, the total number of effective gametes per female $\left(k_{f}\right)$ is the random sum

$$
k_{f}=x_{f_{1}}+x_{f_{2}} \ldots+x_{f} M_{f},
$$

in which each of the $M_{f}$ terms has the same distribution, so that the mean and variance of $k_{f}$ are

and

$$
\varepsilon\left(k_{f}\right)=\varepsilon\left(M_{f}\right) \cdot \varepsilon\left(x_{f}\right)
$$

$$
\operatorname{var}\left(k_{f}\right)=\varepsilon\left(M_{f}\right) \cdot \operatorname{var}\left(x_{f}\right)+\left[\varepsilon\left(x_{f}\right)\right]^{2} \cdot \operatorname{var}\left(M_{f}\right)
$$

The statement for males is analogous.

Assume that the probability of a mating event for an individual, within a time interval of unit length, is sufficiently small, so that the distribution of events is Poissonian. Then for individual males the total number of matings $\left(M_{m}\right)$ in a time interval of length $t$ can also be taken to have a Poisson-distribution (with parameter $\beta t$, say), so that the probability of no mating is

$$
P_{0}(t)=e^{-\beta t} .
$$

However, for individual females it is more realistic to assume that no 
multiple matings occur in such a time interval, which means that $M_{f}$ takes the values 0 or 1 . So $M_{f}$ is binomially distributed with parameter $\theta$, say. Notice that $\theta=1-P_{0}(t)$. Thus, considering any time interval from $t=0$, $M_{m}$ has a Poisson-distribution, with parameter $\alpha$, say; whilst $M_{f}$ has a binomial distribution with parameter $\theta$, say.

It is reasonable to assume both $x_{f}$ and $x_{m}$ to follow a Poisson-distribution (parameters $\lambda_{f}$ and $\lambda_{m}$, respectively).

Then, from (4) and (5):

$$
\varepsilon\left(k_{f}\right)=\theta \cdot \lambda_{f} \text { and } \operatorname{var}\left(k_{f}\right)=\theta \cdot \lambda_{f}\left[1+\lambda_{f}(1-\theta)\right] .
$$

Similarly $\varepsilon\left(k_{m}\right)=\alpha \cdot \lambda_{m}$ and $\operatorname{var}\left(k_{m}\right)=\alpha \cdot \lambda_{m}\left(1+\lambda_{m}\right)$.

Since $\theta=\alpha$ and $\theta \cdot \lambda_{f}=\alpha \cdot \lambda_{m}$, because the offspring originates from equal numbers of maternal and paternal gametes, one obtains, by substitution into (2), followed by adding the expressions for males and females (as can be done with independent samples) and, finally writing $\lambda_{f}$ for $\lambda_{m}$ (see above) the expression

$$
\operatorname{var}(\Delta q)=\frac{p q}{4 \mathcal{N}}\left[4+\lambda_{f}(2-\theta)\right]
$$

For constant population size, i.e. $\theta \cdot \lambda_{f}=2$, this reduces to

$$
\operatorname{var}(\Delta q)=\frac{p q}{2 \mathcal{N}}\left(1+\frac{2-\theta}{2 \theta}\right) \geqq 1 \cdot 5 \frac{p q}{2 \mathcal{N}} .
$$

From (2) it follows $\frac{p q}{2 \mathcal{N}_{e}(v)} \geqq 1 \cdot 5 \frac{p q}{2 \mathcal{N}}$, so that $\mathcal{N}_{e}(v) \leqq \frac{2}{3} \mathcal{N}$.

As an illustration enter in (2) the adjusted ratio's var $(k) / \varepsilon(k)$ for adult females (1.82) and males (3.18) from table 1 .

Averaging over males and females as before, gives

$$
\operatorname{var}(\Delta q)=\frac{p q}{4 \mathcal{N}}\left(1+\frac{1 \cdot 82+3 \cdot 18}{2}\right),
$$

so that $\mathcal{N}_{e}(v) \approx 0 \cdot 7 \mathcal{N}$, which actual value agrees well with $\mathcal{N}_{e}(v) \leqq \frac{2}{3} \mathcal{N}$ from our model.

Expressions analogous to expression (6) can be derived for the following situations:

1. Females may mate more than once, but the total number of zygotes per female remains the same.

2. Females may mate more than once, which leads to a higher number of zygotes per female.

However, also in these cases, $\operatorname{var}(k)>\varepsilon(k)$ and $\operatorname{var}(\Delta q)>\frac{p q}{2 \mathcal{N}}$, i.e. $\mathcal{N}_{e}(v)<\mathcal{N}$.

\section{ReFERENGES}

CROW, J. F. 1954. Breeding structure of populations. II. Effective population number. Chapter 43 in Statistics and Mathematics in Biology (ed. Kempthorn et al.). Iowa State College Press, Ames, Iowa.

CRow, J. F., AND Krmura, M. 1970. An Introduction to Population Genetics Theory. Harper and Row, New York, Evanston \& London. 